\title{
Radiology Systems of the Nineties: Meeting the Challenge of Change
}

\author{
Gwilym S. Lodwick and Jaime L. Taaffe
}

\begin{abstract}
Digital imaging technology, particularly reconstructed images such as computed tomography and magnetic resonance imaging, has fueled the increased demand for radiologic services but has intensified storage and communications problems. Today more than $25 \%$ of radiologic examinations are digital in origin and, with progressive replacing of film images by digital images likely through the introduction of imaging plate technology, the radiology profession is undertaking the massive effort of ovolving a new system where digital images will be transmitted, stored, retrieved and displayed by a multicomponent system connected by a local area network. Through this system, images will be nearly instantly accessible to anyone who needs them. A leading hypothesis is that when the volume of digital examinations reaches $50 \%$ of the whole, cost and efficiency considerations will lead to a massive conversion to the digital image management system, which will progress spontaneously. This conversion, unless planned for in today's equipment acquisitions, could lead to great economic stress in hospitals. The $50 \%$ point may be reached by the early 1990 s.
\end{abstract}

KEY WORDS: Computed tomography, picture archiving and communications system, and radiology information system.

D URING THE PAST 30 years, radiology has changed from a narrow discipline located in the basement of the hospital to a dynamic set of interrelated specialties functioning at the heart of medical practice. This change is related to the broadening of the scientific base of radiology. Foremost are the revolutionary improvement in image quality and the speed with which image information can be captured. New contrast agents and selective catheterization have allowed dynamic imaging of vascular organs. New nuclear tracers provide highly sensitive imaging technologies for detecting disease.

From the Departments of Radiology. Massachusetts General Hospital and Spaulding Rehabilitation Hospital, Boston.

Address reprint requests to Gwilym $S$. Lodwick, MD. Department of Radiology, Massachusetts General Hospital, 32 Fruit St, Boston, MA 02114.

An earlier version of this report was published in West $J$ Med 145:848-852, 1986. Reprinted with permission.

(c) 1988 by W.B. Saunders Company

0897-1889/88/0101-0002\$03.00/0
Radiologists now perform tumor biopsies, reopen vessels and drain abscesses.

A truly fundamental advance is the computed construction of computed images, which has brought digital imaging into the clinical environment. Radiology is now more than a medical discipline; it includes physicists, engineers, computer scientists, radiobiologists, and physicians. Given the organizational and interdisciplinary complexity of the modern radiological sciences department, computer-based communications systems are increasingly vital to effective functioning.

The present trend toward the replacement of existing film-based imaging with digital images began with the introduction of computed tomography $(\mathrm{CT})$. This trend is accelerating to the point where $25 \%$ or more of the daily production of images is digital. ${ }^{1}$ While most such digital images are of relatively low information density, $512 \times 512$ pixels $\times 12$ bits or less, a new imaging technology using a solid state plate and laser scanning for providing high resolution digital images promises to sharply increase the volume of digital image production. Using this technology, it becomes possible to display images of high informational content, such as mammography and chest and bone, on high resolution $4 \mathrm{~K} \times 4 \mathrm{~K}$ pixel monochrome displays. ${ }^{2}$ Given the volume of image production in the busy department, if all images were digital, the amount of new digital information that would need to be stored, transmitted, and displayed would be more than four gigabytes per inpatient bed per year. ${ }^{3}$ In fact, each patient's images need to be displayed as many as ten times during the first week of admission. ${ }^{4}$ Realistically, we are only beginning to understand the requirements for the systems that comprise a picture archiving and communications system (PACS), and for this reason, we are not yet ready to implement a computer-based radiology system that can handle the imaging requirements of the large department.

With the volume and proportion of digital images growing steadily without an adequate system for digital image management (PACS), there is pressure to identify all of the problem 
areas and to resolve the issues surrounding digital image management. In the meantime, the profession is obliged to compromise by recording digital images on film. The management of film images has long been a thorny problem, since all who care for the patient both want and need to see the images at the same time. In a department with poor control of the film library, this demand creates a chaotic situation where film images are lost or stolen, followed by a sequence of events that tend to delay, and at worst, cripple the process of taking care of the patient. ${ }^{5}$ If we could reverse this trend and provide digital storage and display of all images, then it would be technologically possible for all physicians to see the same images at the same time, and the single most difficult problem in radiology would be resolved.

\section{THE RADIOLOGY INFORMATION MANAGEMENT SYSTEM}

The problem of the film library is not a new one; it has just become less manageable with increased volume and demand. By 1966, with growing accessibility of computers, new systems for implementing solutions to scheduling, filing, reporting, and billing began to appear. ${ }^{6-11}$ At first, each system seemed to provide its own unique approach to resolving the radiology management problem; some were oriented to methods of getting the consultation reported in a timely fashion, others to getting the bills out. With passage of time it became apparent that certain solutions were better than others, and that some innovations were obviously desirable, so that most systems came to have certain features in common with each other. The system with which Dr Lodwick was associated, Missouri Automated Radiology system (MARS), went on line at the University of Missouri in April 1970, ${ }^{12}$ and underwent constant modification until it became an extremely useful system. In 1974 the cost effectiveness of MARS was the subject of a detailed economic analysis, ${ }^{13}$ following which the hospital assumed financial support of the system. A major element of the continuing success of MARS was that the Massachusetts General Hospital Utility Multi Programming System (MUMPS) programming language permitted on-line modification without interruption of function. Hence, MARS was kept up to date. ${ }^{14}$
However, this implies the presence of a staff of MUMPS programmers available within the department to implement changes and additions to the system. With every systems change, reevaluation of function was necessary. While we were able to correct problems with our own code, problems beyond our control, such as bugs in the vendor supplied MUMPS operating system, were a maddening source of down time. From start up, more than 6 months elapsed before operating system problems were brought under control.

For all systems, rapid turnaround of reports was the problem least easily resolved, ${ }^{15-18}$ and is often a problem today. With MARS, we ultimately provided the radiologist a choice of three methodologies for recording interpretations of the examination. For on-line reporting, one had the option of typing a brief report on the terminal, correcting and signing it on the spot, or creating a telegraphic-styled report from words and phrases selected from an on-line key word in context index. The third alternative was to dictate into an endless belt recorder for secretarial transcription. Because so many of the radiologists who used MARS chose one of the instantaneous recording options, the volume of secretarial transcriptions ranged from $10 \%$ to $50 \%$ of the whole. This resulted in a transcription backlog during the week ranging from on line to an hour or so. Instantaneous reporting provided especially valuable services to the wards on weekends and to the outpatient clinic, where both reports and films were sent to the clinic with the patient. This combination of reporting technologies proved very effective.

The experience of dealing with radiologists who had the option of choosing between dictation and using the computer for reporting confirms that it is not always the young radiologist who is most receptive of new technology, nor the more elderly who is resistive; some radiologists seem to be born with their attitudes set in concrete. However, on the whole, residents who were brought up with MARS were most proficient in its use.

For film tracking, Bauman's use of bar coding has proved successful. ${ }^{19}$

It is true that a well-designed and supported RIS becomes an instrument of management poli- 
cy. It is equally true that to be successful, a Radiology Information System (RIS) must have the support of the chief of the department of radiology, and in a hospital, of the director of the hospital. A personal anecdote provides an interesting reflection on the value of the RIS. After more than 20 years as chair of the department of radiology at the University of Missouri, I (Dr Lodwick) decided that I had achieved about all that I could as manager and retired to research and writing. My successor apparently ran into some of the insoluble issues that had plagued me during my tenure, and ultimately gave it up. Not without some concessions by the dean and the hospital, I took on the responsibility again, and was pleased to find that within a week it was possible to generate 5-year charts showing patient volume, mix, financial trends, and time flow-in short all information necessary to resume planning for the department's future. In my absence, the computer had been quietly ticking along, "faithfully" collecting all of this information which simply awaited analysis. I do not see how a large department can be effectively managed without a RIS.

Lehr, who redesigned MARS for the University of Chicago, ${ }^{20}$ has written comprehensively on RIS. $^{21}$ The formation of the RIS Consortium ${ }^{22}$ has led to a systematic implementation of DECRAD, a state of the art information system, which is being widely implemented. Many other RIS are now being marketed. Selecting a radiology information system can be one of the most important decisions made by a radiology department. For the department manager about to make this decision, the report by Lehr and Steinberg will help to assure success. ${ }^{21}$ One fact is certain: to attempt a Procrustean fitting of the department of radiology to an information system of inflexible design is a path to assure failure.

\section{THE RADIOLOGY IMAGE INFORMATION SYSTEM (PACS)}

With the initiation of digital images into the clinical environment and with successful RIS as the model, we began to examine the possibility of implementing an image information management system. ${ }^{23}$ By 1977 , digital image management had become sufficiently a problem that Hill et al were implementing data compression for more efficient archiving of CT findings. ${ }^{24} \mathrm{~A}$ major stimulus for giving serious consideration to such digital systems has been the promise of mass storage devices for archiving digital data, particularly the digital optical disc. This festering problem of storing voluminous digital image files without systematic access added a sense of urgency to planning and implementation of the new image management system. Implementation has been slow for many reasons, a major one being that industry is still not geared to the task of producing a system for storing, retrieving, transmitting, and displaying digitally acquired radiographic images within the constraints of speed, spatial resolution, and human engineering imposed by the radiologist users. At the same time, radiologists have not had hard data to answer many questions related to the design specifications of the proposed system. Ultimately, many questions will be resolved by research with human subjects, some others by trial and error, and all ultimately by the market place.

The design and implementation of the image information management system is a massive scientific effort. The successful implementation will have an impact far beyond the perimeters of radiology. Accordingly, as a systems problem it will not be rapidly resolved, but rather, success will depend on simultaneously solving tough technical problems now under intensive study. Many of these are beyond the normal expertise of radiologists, while others can be solved only with the professional guidance and collaboration of radiologists. Recently, a number of review articles have illuminated the technical scope of the image management system, and the progress that has been made. ${ }^{25-27}$ Several problem areas are briefly discussed below.

\section{THE FINANCIAL ATMOSPHERE}

The desire to contain costs and the change in methods of radiology reimbursement to charging costs against a total predetermined by federally imposed diagnostic related groups (DRG) has placed us in the position of needing to design a system that diminishes operating costs. The economics of PACS have been examined, ${ }^{28,29}$ but as yet, we do not have a good enough model to show that PACs can compete economically with current film systems. We have not been able to afford the implementation of those models of 
systems that incur major costs for displays. Yet, digital imaging continues to grow in volume, and regardless of cost, the need for new systems to replace outmoded technology is pressing.

As of now, several systems for creating digital $x$-rays are being extensively tested, based on the Fuji technology, which acquires images digitally from plates but captures the digital information on film. This technology permits bypassing the costs of digital storage, networking, and displays. Such systems are only one component of the image management system but can be plugged into an existing department without requiring a major change in the radiologist's routine, and possibly without major change in operations or costs. While these systems tend to prepetuate film based departments, they satisfy the traditional demand for film images. The Fuji plate technology and other wide area technologies permitting the generation of high resolution digital images will accelerate the conversion to the digital department.

\section{THE ACR-NEMA DIGITAL INTERFACE COMMUNICATIONS STANDARD}

The design specifications of most digital imaging equipment do not include standardized output signals. The result of this is that if image signals are to be transmitted on a network, or displayed on another manufacturers equipment, a special interface must be designed to make the original image signal interpretable. In a major departure from the past, the users of radiological imaging equipment, represented by the American College of Radiology (ACR), and the manufacturers of imaging equipment, represented by the National Electrical Manufacturers Association (NEMA), have formed a joint committee to specify a standard interface for the transfer of digitally formatted images. In a period of 2 years, this committee and its working groups have created an industry standard interface, ACR-NEMA Digital Imaging and Communications Standard, Publication No. 300-1985, available from the National Electrical Manufacturers Association, 2101 L St NW, Sutie 300, Washington, DC 20037..$^{30}$ The interface specifies the lower four layers of the International Standards Organization-Open Systems Interface sevenlayer reference model. ${ }^{31}$ The standard specifies a 16-bit parallel interface with six control signals.
Data rates of 8 megabytes can be achieved over a cable of $15 \mathrm{~m}$ maximum length. The standard is now being implemented by various manufacturers and other interested parties.

Working Group 4 of the ACR-NEMA Committee has submitted the draft of a Magnetic Tape Standard for review and approval. This draft describes a standard for exchange media. Working Group 5 has identified the compression methods likely to be used in medical imaging for inclusion in the ACR-NEMA Compression Standard, and for each method, specified the data elements and their ordering sufficiently that, based only on information from the Standard, it is feasible to design a decorder that will properly reconstruct the image from the data sent by any encoder conforming to the Standard.

With feedback from those who have implemented the 1985 Communications Standard, Working Group 6 has proposed the revised ACR-NEMA Standards Publication 300-1988, Version 2.0 ACR-NEMA Digital Imaging and Communications Standard. It is expected that this revision and the two new standards will be approved and published during 1988.

Working Group 8 is now formulating its approach to developing a standard interface for communications between RIS, PACS, and the hospital information system (HIS). ACRNEMA Committee will continue its function for the forseeable future, upgrading and creating new standards as required.

\section{THE DIGITAL WORKPLACE}

Of all the elements of a digital image management system, the display is perceived as most problematic. This is because the display must be designed to replace not just a set of illuminated view boxes, but rather a complex system for efficiently presenting a pre-programmed day of work for radiologists, with as many images as necessary for thoroughly understanding each case. The rule is that the display must function at least as well as the radiologist's present equipment, or it will not be acceptable. Radiologists must be able to interrupt their examination of specific cases, look at images of immediate interest to referring physicians, and easily resume their scanning of those cases. Images that are upside down or backward must be repositioned. 
Images must move rapidly and with precision: it must be possible to reposition images in closely aligned groups for ease of comparison. It should be possible to window CT scans, or to call up images from another examination for comparison.

The display must be a facile window into the image file of the institution. It must be possible to simultaneously review previous reports on the case under study, or to annotate examinations where desirable. It should be possible to accomplish all of these maneuvers at a display screen time of $<0.5$ seconds, or at a rate comparable to moving films by hand. Further, it should be possible to obtain hard copies on film or paper.

The largest and most elaborate workstation displays will need to present images at $2,048 \times$ 2,048 square pixels with 12 bits of grey-scale depth. Cox et al believe that a single display screen may suffice, ${ }^{26}$ - a debatable issue. Less elaborate displays may have less spatial resolution but can accomodate by reviewing smaller areas of images of higher pixel densities and through zooming. So far, displays which fully meet the needs of radiology have not yet been marketed, and the prototype models have been prohibitively expensive. A generic display with the features of a high level work center, versatility, and more is under development in the radiology department at Massachusetts General Hospital (R-Star). Many others are also under development.

\section{HEAD-END OR CENTRAL SERVICES}

Initially it would seem that a PAC system is comprised solely of a work station, an image archive, and a communicating local area network between them. This model is a fine starting place to begin PACS research and development, but it is too simplistic and leaves out many services and functions such as arbitrating conflicting requests from the work stations, coordinating the acquisition and filing of new cases, providing services ill-suited to the work stations, and performing system integrity and recovery functions. A few examples will clarify the server's role.

It is obvious that the PAC system must require some form of password before allowing users access to work station functions. Not so obvious is the necessity for a finer grained access mecha- nism. For example, radiology residents may have access to most cases but may not have the ability to alter the images or reports, whereas staff may. It may be that only senior staff will have the privilege to enter cases into the teaching files (compilations of interesting cases). Furthermore, as computer networks become more pervasive and play centrally in the department's activities, it may become prudent to maintain audit trails and logs of activity to insure the integrity of the system.

The acquisition and filing of new exams, even with ACR-NEMA devices, into the PACS database is a nontrivial problem. The exams must be checked for consistency, that is, missing or contradictory data, and converted into a format compatible with the PAC system. The image data may be compressed, possibly requiring specialized hardware.

The image archive may be the largest in terms of storage size, but it is one of the simplest. The database that describes the patients and all their associated exams is far more complex when one considers that complex queries may be supported (relational multi-key) and that the database may have to track exam focations on many different storage classes (on line magnetic disk, optical disk, off line tape). Other databases might include active radiologists and their current primary responsibilities, current disposition of new cases, and viewer preference for exam presentation. If exams may be viewed at any work station, then the system is simplified if these databases and the software that operates upon them are in a logically central location. In addition, the backup/restore operations might be best performed at a centrally located site.

For the PAC system to be integrated into the department's operation, it becomes necessary for the system to exchange data with other computers within the department (RIS), and the hospital (medical records, pathology). Since data conversion and queued access may be required in communicating with these other systems, it becomes easier to create processes on the server that handles these requests. We see that not only must patient exams be entered into the system, but users of the PAC system may well wish for exams (text as well as image data) to be exported out from the PAC system to other computers. As 
the users of the work station become more proficient with computers in general, they may wish to avail themselves of the wide-area computer network linking systems spanning the globe such as the Internet, Bitnet, UUCP, etc. Again, these functions might or might not be best performed on a centralized server supporting the work stations.

As evident in any radiology department's budget, the need for hardcopy output, films, slides, and prints, is high. Thus, a PAC system must ultimately include film recorders and/or slide makers. Certainly these devices should be shared by all the work stations. As the role of PAC systems becomes more critical to the department, it becomes vital that the integrity of the system be established and continually verified. In addition, if hardware or software failures should occur, it is paramount that the system should try to correct or minimize the resultant errors. Again, these functions are simpler if performed centrally. As pointed out in the last few paragraphs, some form of server needs to be provided to handle the needs of the work stations as well as to maintain the various databases.

Though it may appear that the best way to accomplish this is to have at the center of the system one large computer, it may be advantageous to distribute these server functions across many smaller computers for reasons of reliability and cost. Nevertheless, the notion of a logically central server may be the best way to coordinate the entire PAC system.

\section{WORK-FLOW MANAGEMENT AND CENTRAL-SERVICES ISSUES}

The literature abounds with statements of how PAC and RIS systems will, and must, become integrated. A simple model of this interaction is where the RIS tells the PAC system what cases to expect, and upon viewing, the PAC system then informs the RIS of its completion. Important functions, not so evident, must be addressed. For example, must the radiologist explicitly search for and call down each exam to be viewed or might the PAC system know each radiologist's responsibilities, and thus, automatically make these cases available to him? Does the PAC system alert the radiologist who tries to view a case that is currently being viewed at another work station, of this fact so as to prevent two reports from being rendered? Will the PACS attempt to choose the appropriate comparison exams for each new case to be viewed?

\section{DATA COMPRESSION}

Give the high data rates and the huge storage capacities required for archiving and rapid transfer of digital images, methods for reducing data volume are of great interest. Each digital image contains information that is redundant in that it does not contribute to the diagnostic content of the image. Some images contain a great deal more redundant information than others. Contrast level redundancy refers to patterns of repetition in the degree of pixel brightness. Huffman encoding is a commonly used method of providing contrast compression through estimating the probability of occurrence of pixel contrast values. ${ }^{32}$ Lossless encoding permits image data compression which is not subject to loss of information on image decompression. However, the amount of image compression that can be achieved without loss of information is relatively small, such as $2: 1$ or $3: 1$. Other compression techniques extend to $20: 1$ or higher, but with nonrecoverable loss of image information. Image decompression times are also a significant issue, since the time required for decompression may be many times longer than that required for the initial compression.

At this point, if images can tolerate compression at high rates without loss of diagnostic quality, this would make the image transmission, archiving and retrieval times much faster and less expensive. Because of this economy, image data compression has become an important issue, but one that can only be finally solved by experimentally determining which algorithms can provide the greatest time and cost benefits without significant degradation of diagnostic quality.

\section{THE LOCAL AREA NETWORK}

Computer networks are an essential part of the digital image management system. The twisted wire networks are far from fast enough for image transmission purposes, and coaxial cable provides a marginal top signaling rate of $60 \mathrm{mega}-$ bits per second. ${ }^{26}$ It seems likely that future transmission needs will be met only through 
some other means such as the use of fiber optic cables, which will provide a signaling rate of more than 200 megabits per second. While few departments are now using networks for image transmission, many are installing cables, often both coaxial and fiber optic cables.

It should be noted that the data signaling rate is much higher than the actual data throughput rate, the difference due to overhead such as the size of records being transmitted, the size of buffers, and the number of active nodes on the network. ${ }^{26}$ Obviously, image data compression is an extremely important variable in improving the data throughput rate of PACS networks.

\section{ARCHIVING AND DATA BASE MANAGEMENT}

In radiology today, digital images are stored on magnetic tapes and discs, usually in files which are often remote from the active department. Film copies of digital images, such as CT, magnetic resonance imaging, and ultrasound, are filed in the master folder in the film library. If the film copies of CT images are lost, the magnetic media are withdrawn from storage, and are scheduled into the patient queue for access to the CT scanner, where they are rephotographed. This process takes 24 hours or longer under most circumstances. Archiving of digital images on mass storage media such as optical disc or tape storage is clearly desirable, but in order for mass storage to work, there must be mechanisms, and demands, for accessing mass storage. There must be data transmission networks, imaging devices that feed the transmission networks, and there must be work stations for accessing and displaying the digital images which are filed in mass storage.

We have a chicken and the egg situation; you can't have a part without the whole. These systems are generally not available except as prototypes. Noz et al recently surveyed PACS systems, largely in universities and in large hospitals, to find that some prototype systems are centralized and others decentralized. ${ }^{25}$

In a centralized system, every image that goes onto the network gets sent to the image archiver/file server. All requests also go through the central archive. The decentralized system may have local magnetic disc archives and a ring or star configuration, so that images and messages can be sent directly to other points on the network without going through a central archive. One commercial system offers two digital optical disc archives featuring multiplayer laser optical disc "jukeboxes." These disc stores have a foursecond response time to search, load, and spin to the image. This system is based on a centralized topology. Generally, however, massive storage is still a part of the ongoing prototype development. Data base management systems are a part of this development.

\section{DISCUSSION}

Those of us who have been participating in the development of the radiology image management system can see that all of the parts of the system will inevitably be assembled. When will we fit them all together and start using them as a system? We can see that this is now happening in a limited way, largely in university settings using manufacturer's prototypes. The manufacturers of imaging equipment have assembled PACS research and development teams and are looking at the marketplace, measuring the radiologist's enthusiasm, seeking opinions from the experts, and hedging their bets on the future.

PACS is big money. The well-informed guess is that the image information system will move off center and gain momentum when the proportion of digital images to film images reaches $50 \%$. As of 1984 , the evidence is that the volume of digital imaging at Massachusetts General Hospital was $20 \%$ of the whole; plain film images represented the remaining $80 \% .^{3}$ This would indicate that $30 \%$ of film images would need to be converted to digital format before the system would begin to take off.

As of now, the major viable large volume source of high resolution digital images which would replace standard $\mathrm{x}$-ray images would appear to be from the clones of the Fuji imaging plate system now being produced in Japan and Europe. If this proposed algorithm of $50 \%$ replacement is correct, we will know when the time is to come by watching the sales of imaging plate technology. However, money must be available for health care institutions to buy such technology, which means that there will need to be enough savings to make these systems attractive. Given this scenario, it can be anticipated that radiology will be well into the 1990s before operational image management systems are a 
significant factor in improving the quality of radiological practice. In the meantime, the component systems will be polished, and with current trends, the prices will diminish.

\section{CONCLUSIONS}

After nearly 20 years of development, radiology information systems are now routinely used in many departments. Still incompletely resolved is the reporting function, where the equivalent of direct dictation and transcription is desirable. Voice recognition has been tested but not yet proved sufficiently reliable. ${ }^{33}$ Coded reports, direct entry by radiologists, telephone access to dictated reports, and tightly coupled transcription pools are all solutions which await a better one.

What is to be the future of the RIS in the presence of the PACS system? RIS covers a broad spectrum of functions which support but which are not a direct part of image management. Lehr believes that the implementation of PACS should probably be considered as a replacement of the film file management module of a RIS.$^{34}$ PACS could not survive in an environ- ment without the RIS function. The evolution of PACS will inevitably include the fusion of the two systems.

The key to the future implementation PACS appears to depend on the implementation in the radiology department of a cost effective digital replacement of the standard $x$-ray image. It can be predicted that radiologists will give up film images with great reluctance. For this reason the systems which are based on the Fuji imaging plate principle, producing either a digital $x$-ray or alternatively, an image on a high resolution screen appear to be the most viable candidate for replacing the standard $x$-ray.

Economics will remain a major issue. If the imaging system can be demonstrated to speed the process of inpatient care, save money by providing efficiencies in film production and management, and particularly, if systems are interfaced with hospital systems, and can provide insight to hospital administrators about how radiological resources are used, then money will be found to support their purchase. It is not so much whether society can afford information management systems, as whether society can afford not to have them.

\section{REFERENCES}

1. Bauman RA, Lodwick GS, Taveras JM: The digital computer in medical imaging: A critical review. Radiology 153:73-75, 1984

2. Manipulating digital $x$-rays enhances different features. Electronics 39-41, February 3, 1986

3. Goodsitt MM, Bauman RA, Lodwick GS: Digital workload in a large radiology department. Proceedings of the Society of Photo-optical Instrumentation Engineering (SPIE) vol 626, Medicine XIV/PACS IV, 1986

4. Templeton AW, Dwyer SJ III, Johnson JA, et al: An on-line digital image management system. Radiology 152:321, 1984

5. Lodwick GS: Pictorial information systems and radiology: Improving the quality of communications, in Hohne KH (ed): Pictorial Information Systems in Medicine, vol 19. NATO Advanced Sciences Institute (ASI), Series F. Berlin, Springer-Verlag, 1984 Series F: Computer and Systems Sciences 1984

6. Kricheff II, Korein J: Computer processing of narrative data: Progress and problems, in Proceedings of the First Conference on the Use of Computers in Radiology. Columbia, Department of Radiology, University of Missouri, October, 1966, pp D18-D33

7. Templeton AW, Lodwick GS, Sides S, et al: Radiate: A radiology and hospital computer oriented communicating system, in Proceedings of the First Conference on the Use of Computers in Radiology. Columbia, MO, Department of
Radiology, University of Missouri, October 1966, pp D44D50

8. Barnhard HJ, Long JM: Automatic coding and manipulating of radiology diagnostic reports, in Proceedings of the First Conference on the Use of Computers Radiology Columbia, MO, Department of Radiology, University of Missouri, October 1966, pp D51-D62

9. Lamson BG: Storage and retrieval of medical diagnostic statements in full English text, in Proceedings of the First Conference on the Use of Computers in Radiology. Columbia, MO, Department of Radiology, University of Missouri, October 1986, pp D34-D43

10. Lindberg DAB, Schroeder JJ, Rowland LR: Acquisition and utilization of hospital data using a computer system, in Proceedings of the First Conference on the Use of Computers in Radiology. Columbia, MO, Department of Radiology, University of Missouri, October 1966, pp D63-D72

11. Brolin I: Automatic typing and transmitting of radiological reports, in Proceedings of the First Conference on the Use of Computers in Radiology. Columbia, MO, Department of Radiology, University of Missouri, October 1966, D3D17

12. Lehr JL, Lodwick GS, Reichertz P, et al: Experience with an on-line reporting system, in Proceedings of the Second Conference of Computer Applications in Radiology. Washington, DC, US Department of Health, Education and 
Welfare, Food and Drug Administration, September 1970 , pp 401-408

13. Dickhaus EA: Economic evaluation of Missouri automated radiology system. MARS-A case study. Doctoral Thesis University of Missouri, Columbia, MO, May 1974

14. Lodwick GS, Wickizer CR, Dickhaus E: MARS-Its tenth anniversary of operation and its future. Methods Inf Med 19:125-132, 1980

15. Bauman RA, Poitras JW, Pendergrass HP, et al: Computer-entered radiographic reports: The MGH approach, in Proceedings of the Third Conference on Computer Applications in Radiology. Columbia, MO, Department of Radiology, University of Missouri, September 1972, pp 6874

16. Leeming BWA, Simon M: Computerized radiologic reporting-The clip system, in Proceedings of the Sixth Conference on Computer Applications in Radiology \& Computer/Aided Analysis of Radiological Images. Washington, DC, Institute of Electric and Electronic Engineers (IEEE) Computer Society, June 1979, pp 414-419

17. Mani RL: Raport radiology systems: Results of clinical trials. AJR 127:811-816, November 1976

18. Wheeler PS, Wimborg DW, Gitlin JN: The Johns Hopkins radiology reporting system. Radiology 119:315-319, 1976

19. Bauman RA, Arenson RL, Barnett GO: Computerbased master folder tracking and automated file operations, in Proceedings of the Fourth Conference on Computer Applications in Radiology. Reston, VA American College of Radiology, March 1975, pp 469-480

20. Lehr JL: Installation of MARS at the University of Chicago, in Proceedings of the Eighth Conference on Computer Applications in Radiology. Reston, VA American College of Radiology, May 1984, pp 35-45

21. Lehr JL, Steinberg FL: The radiology information system, its evolution and current status. Crit Rev Med Inform 1:259-309, 1987

22. Arenson RL, Gitlin JN, London JW, et al: The formation of a radiology computer consortium, in Proceedings of the Seventh Conference on Computer Applications in
Radiology. Reston, VA American College of Radiology, April 1982, pp 153-164

23. Lodwick GS: Better interpretation and reporting of radiant images. MEDINFO 77 Proceedings, New York, North-Holland, August 1977, pp 575-583

24. Hill RL, Jost RG, Evens, RG: Data compression for computed tomography. Congresso Internacional de Radiologica, Rio de Janeiro, Brazil, October 23-29, 1977

25. Noz ME, McGuire GO Jr, Erdman WA: Local area networks in an imaging environment. Crit Rev Med Inform 1:81-133, 1986

26. Cox GG, Dwyer SJ III, Templeton AW: Computer networks for image management in radiology. An overview. Crit Rev Diagn Imag 25:333-371, 1986

27. Pictorial information systems in medicine. NATO ASI Series, Series F, Computer and Systems Sciences, vol 19. Berlin, Springer-Verlag, August 27-September 24, 1984

28. Bell HS, Enzmann DR: A computerized economic model for a radiology department considering PACS, in Proceedings of the Eighth Conference on Computer Applications in Radiology. Reston, VA American College of Radiology, May 1984, pp 463-469

29. Dwyer SJ, Templeton AW, Martin NL, et al: The cost of managing digital diagnostic images. Radiology 144:313318,1982

30. National Electrical Manufacturers Association: ACR-NEMA digital imaging and communications standard. Washington, DC, NEMA, publication no. 300-1985

31. Folts HC, des Jardins R (eds): Open systems interconnection (OSI) - Standard architecture and protocols, in Proceedings of the IEEE. Washington, DC, IEEE Computer Society, 1983

32. Huffman DA: A method for the construction of minimum redundancy codes. Proceedings of the Institute of Radio Engineers (IRE) 40:1098-1101, 1952

33. Leeming BW, Porter D, Jackson JD, et al: Computerized radiologic reporting with voice data-entry. Radiology 138:585-588, 1981

34. Lehr JL: Impact of manual and computer assisted PACS for Automated PACS. Proc SPIE 418:6-13, 1983 